\title{
Maxillary and mandibular first molar with five root canals diagnosed with cone-beam computed tomography: A review and two case reports
}

\author{
Dr. Keyur P. Chande ${ }^{1}$, Dr. Narendra U. Manwar ${ }^{2}$, Dr. Manoj G. Chandak ${ }^{3}$, \\ Dr. Jyoti Lokade ${ }^{4}$ \\ ${ }^{1}$ (Post-Graduate Student,Dept. Of Conservative Dentistry And Endodontics Sharad Pawar Dental \\ College,Datta Meghe Institue Of Medical Sciences (Du).Wardha, India) \\ ${ }^{2}$ (Professor, Dept. Of Conservative Dentistry And Endodontics Sharad Pawar Dental College,Datta Meghe \\ Institue Of Medical Sciences (Du).Wardha, India) \\ ${ }^{3}$ (Professor, Dept. Of Conservative Dentistry And Endodontics Sharad Pawar Dental College,Datta Meghe \\ Institue Of Medical Sciences (Du).Wardha, India) \\ ${ }^{4}$ (Reader, Dept. Of Conservative Dentistry And Endodontics Sharad Pawar Dental College,Datta Meghe \\ Institue Of Medical Sciences (Du).Wardha, India)
}

\begin{abstract}
Thorough knowledge of root canal morphology is essential for the success of endodontic treatment. There are rare aberrant, variations in canal number and configuration in maxillary and mandibular molars, which could affect treatment outcome. This paper presents the endodontic management of a maxillary first molar with two palatal canals and three buccal canals and mandibular first molar with two mesial and three distal canals. In this paper this morphology was confirmed using cone-beam computed tomography (CBCT) and surgical operating microscope. CBCT can provide dentists with high quality 3-dimensionals images of dental structure due to it's high spatial resolution. This paper is intended to reinforce clinician's awareness of the rare morphology of root canals
\end{abstract}

Keywords: cone-beam computed tomography scanning, five root canals, maxillary and mandibular first molar.

\section{Introduction:}

Knowledge of both normal and abnormal anatomy of the root canal system dictates the parameters for execution of root canal therapy and can directly affect the outcome of the endodontic therapy ${ }^{1}$. It is highly imperative that the clinician should be aware of possible morphological variations of teeth for successful endodontic therapy. There are numerous variations in the canal number and configuration in maxillary molars ${ }^{2}$. The root canal anatomy of maxillary first molars has been described as three roots with three canals, and the commonest variation is the presence of a second mesiobuccal canal(18\% and $96.1 \%)^{1,2}$. Other variations include one, four, and five roots and unusual morphology of root canal systems within individual roots. Case reports with five and six root canals or with a C-shaped canal configuration have also been reported earlier. Six root canals with two mesiobuccal, two distobuccal, and two palatal have been reported ${ }^{3}$. Maggiore et al reported the maxillary first molar having six canals with two mesiobuccal, three palatal, and one distobuccal ${ }^{4}$, whereas Adanir reported a clinical case having four roots (mesiobuccal, mesiopalatal, distobuccal, and palatal) and six canals with one mesiobuccal, two mesiopalatal, two distobuccal, and one palatal ${ }^{5}$.

A comprehensive review of the root and root canal morphology of the human permanent maxillary first molar ${ }^{6}$. Limited number of cases with 2 palatal roots/canals (Table 1) ${ }^{7-11}$. The incidence of two root canals in the palatal root of maxillary molars has been reported to be $2 \%$ to $5.1 \%$.

The anatomical configuration and the number of root canals of mandibular molars have been investigated in several in vitro and in-vivo studies ${ }^{12}$.

They reported 29 teeth with five root canals in a sample of 2362 mandibular permanent molars. FabraCampos studied 145 mandibular first molars and found that $2.75 \%$ of the teeth had five canals ${ }^{13}$. Radiographic examination on extracted teeth reported mandibular first molars had three distal canals in $1.7 \%$ of the specimens (Fabra- Campos et. al 1985).The present case report discusses the successful endodontic management of a maxillary first molar presenting with three roots and seven root canals. This unusual morphology was confirmed with the help of cone beam computerized tomography (CBCT) scans. 


\section{CASE 1}

\section{Case Report}

A 37-year-old man presented to the Speciality clinics of Department of Conservative Dentistry and Endodontics, Sharad pawar dental college, with the chief complaint of spontaneous Pain in upper right region of the jaw since 2 days. The pain aggravated by thermal stimuli and on mastication. History revealed intermittent pain in the same tooth with hot and cold stimuli for past 1 month. The patient's medical history was noncontributory. A clinical examination revealed a carious maxillary right first molar (tooth 16), which was tender to percussion. Palpation of the buccal and palatal aspect of the tooth did not reveal any tenderness. The tooth was not mobile and periodontal probing depth around the tooth was within physiological limits.

Vitality testing of the involved tooth with dry ice (R C Ice; Prime Dental Products Pvt Ltd, Mumbai, India) caused an intense lingering pain, whereas electronic pulp stimulation (Parkel Electronics Division, Farmingdale, NY) caused a premature response.

Neither any fistulae nor any edema was present.

A preoperative radiograph revealed mesio-occlusal radiolucency, approaching the pulp space with periodontal ligament space widening in relation to the mesiobuccal root (Fig. a). From the clinical and radiographic findings, a diagnosis of symptomatic acute apical periodontitis was made and endodontic treatment was suggested to the patient. Radiographic evaluation of the involved tooth did not indicate any variation in the canal anatomy (Fig. a)

The tooth was anesthetized with $1.8 \mathrm{~mL}(30 \mathrm{mg}) 2 \%$ lignocaine containing 1:200,000 epinephrine (Xylocaine; AstraZeneca Pharma Ind Ltd, Bangalore, India.) followed by rubber dam isolation. An endodontic access cavity was established. Clinical examination with a DG-16 endodontic explorer (Hu-Friedy, Chicago, IL) and surgical operating microscope (Seiler Revelation, St Louis, MO)revealed two canal openings in mesiobuccal, one distobuccal, and two in palatal root. Coronal flaring was done with a nickel-titanium ProTaper series orifice shaper Sx (Dentsply Maillefer, Ballaigues, Switzerland) to improve the straight-line access(Fig. b). The working length was determined with the help of an apex locator (Root ZX; Morita, Tokyo, Japan) and later confirmed using a radiograph. Multiple working length radiographs were taken at different angulations (Fig.c). However, the radiographs did not clearly reveal the number and morphology of root canal systems.It was confirmed by CBCT imaging of the tooth.( Fig.1.h-1.m)

Access cavity was sealed with IRM cement (Dentsply De Trey GmbH, Konstanz, Germany).

An informed consent was obtained from the patient, and a multislice CBCT scan of the maxilla was performed (Kodak $90003 \mathrm{D}$ ) with a tube voltage of $100 \mathrm{KV}$ and a tube current of $8 \mathrm{~mA}$.

The involved tooth was focused, and the morphology was obtained in transverse, axial, and sagittal sections of 0.5 -mm thickness. CBCT scan slices revealed five canals (two mesiobuccal, two palatal, and one distobuccal) in the right maxillary first molar (Fig.1.h-1.m).

At the second appointment, the patient was asymptomatic. Cleaning and shaping was performed under rubber dam isolation using ProTaper nickel-titanium rotary instruments (Dentsply Maillefer) with a crowndown technique.

Irrigation was performed using normal saline, 2.5\% sodium hypochlorite solution, and 17\% EDTA. Mastercone radiograph was taken .(Fig.d)The canals were dried with absorbent points (Dentsply Maillefer), and obturation was performed using ISO standardized 6\% gutta-percha (Dentsply Maillefer) and AH Plus resin sealer (Maillefer, Dentsply, Konstanz, Germany). (Fig.e).

The tooth was then restored with silver amalgam. The patient was advised a full-coverage porcelain fused metal crown and it was asymptomatic during the follow-up period of 6 months and 12 months.( Fig. 1.f,1.g)

\section{CASE 2}

A 37-year-old man presented to the Speciality clinics of Department of Conservative Dentistry and Endodontics, Sharad pawar dental college, with the chief complaint of spontaneous Pain in lower left region of the jaw since 2 days. The patient presented severe lingering pain to cold water applied to the isolated tooth 36.(Fig.2.a) Tooth was non-responsive on percussion and palpation. After administering local anesthesia, and thorough isolation all carious tissue were removed, and an adequate endodontic access was made.

Clinical examination with a DG-16 endodontic explorer (Hu-Friedy, Chicago, IL) and surgical operating microscope (Seiler Revelation, St Louis, MO) revealed the pulp chamber floor showed five orifices corresponding to five root canals: mesiobuccal(MB),mesiolingual (ML), distobuccal(DB), and distolingual(DL) and middle distal(MD) (Fig.b) Working lengths were estimated by using an electronic apex locator (Root ZX; Morita, Tokyo, Japan). Multiple working length radiographs were taken at different angulations (Fig.2.b ). 
However, the radiographs did not clearly reveal the number and morphology of root canal systems. To confirm this unusual morphology, it was decided to perform CBCT imaging of the tooth no.36.( Fig.2.h-2.k )

Access cavity was sealed with IRM cement (Dentsply De Trey GmbH, Konstanz, Germany).

$\mathrm{An}$ informed consent was obtained from the patient, and a multislice CBCT scan of the maxilla was performed (Kodak 9000 3D) with a tube voltage of $100 \mathrm{KV}$ and a tube current of $8 \mathrm{~mA}$. The involved tooth was focused, and the morphology was obtained in transverse, axial, and sagittal sections of 0.5 -mm thickness. CBCT scan slices revealed five canals (mesiobuccal(MB), mesiolingual (ML), distobuccal(DB), and distolingual(DL) and middle distal(MD) in the left mandibular first molar (Fig.2.h-2.k). CBCT images provided valuable information regarding the canal configuration and confirmed the five canals that were not clearly seen in the conventional radiograph.

All canals were cleaned and shaped with Protaper rotary instruments (Dentsply-Maillefer, Ballaigues, Switzerland) under copious irrigation with $5 \%$ sodium hypochlorite. After preparation, the gutta-percha cones were inserted into the root canals and working length was reconfirmed (Fig.2.d ). All canals including the middle distal canal were obturated with gutta-percha and sealer (AH-plus, Dentsply-Maillefer) (Fig.2.e).

The patient experienced no post treatment discomfort and was subsequently referred for appropriate coronal restoration during the follow-up period of 6 months and 12 months.( Fig.f,g)

\section{Discussion}

Several anatomic variations of the configuration of the maxillary first molar have been reported in the scientific literature. The majority of endodontic literature describes the maxillary first molar as having 3 roots with 3 or 4 root canals. 34 laboratorial and clinical studies identify one canal (MB) in $43.1 \%$ of the cases and two canals (MB and MP) in 56.8\%.

The incidence of one apical foramen in the mesiobuccal root was $61.6 \%$. combined sample of 2576 roots from 14 laboratorial and clinical studies of distobuccal root revealed incidence of a single canal (DB) was $98.3 \%$ and the presence of two canals (DB and DP) in $1.7 \%$ of the cases respectively of the cases with one apical foramen of $98 \%$.(Cleghorn et al. 2006).

Caliskan et al. stated that a second canal is found in $65 \%$ of mesiobuccal roots of maxillary first molars ${ }^{14}$. Bond et al. reported a case of a maxillary first molar with six canals of which two canals were in distobuccal root and two canals in palatal root ${ }^{15}$. Three cases of maxillary first molar with six canals, three in mesiobuccal root, two in distobuccal root and one in palatal root have been reorted. (Martınez-Berna'A et al.1983). The prevalence of maxillary first molars with 2 palatal canals is rare. Likewise mandibular first molar also shows variation in normal root canal anatomy. Endodontic management of a mandibular first molar in a patient of Indian origin, having three distal canals within a single distal root has been described ${ }^{16}$.

A mandibular first molar with three distal canals was first reported by Berthiaume et al. however three distal canals ended in separate apical foramina,have also been described ${ }^{17}$.

Quackenbush et al. reported three canals in two extracted mandibular first molars ${ }^{18}$. In this case 2, the distal root which root three separate canals ended in one apical foramina.

Matherne et al. investigated the use of CBCT scanning in identifying root canal systems and compared it with images obtained by using digital radiography. They concluded that CBCT images always resulted in the identification of greater number of root canal systems than digital images.CBCT scanning is a relatively newer diagnostic imaging modality that has been used in endodontics for the effective evaluation of the root canal morphology ${ }^{19}$. Additionally, CBCT technology aids in the diagnosis of endodontic pathosis, assessing root and alveolar fractures, analysis of resorptive lesions, identification of pathosis of nonendodontic origin, and presurgical assessment before root-end surgery.

Baratto Filho et al evaluated the internal morphology of maxillary first molars by ex vivo and clinical assessments using operating microscope and CBCT scanning and concluded that an operating microscope and CBCT scanning were important for locating and identifying root canals, and CBCT scanning can be used as a good method for initial identification of maxillary first molar internal morphology ${ }^{20}$.

The major advantages of CBCT scanning over the conventional CT scans are x-ray beam limitation, rapid scan time, and effective dose reduction; x-ray beam limitation is achieved by reducing the size of the irradiated area by collimation of the primary $\mathrm{x}$-ray beam to the area of interest.

In the present case, $\mathrm{CBCT}$ scanning was used for a better understanding of the complex root canal anatomy. CBCT axial images confirmed the presence of three roots and five root canals, namely mesiobuccall $\left(\mathrm{MB}^{1}\right)$, mesiobuccal $2\left(\mathrm{MB}^{2}\right)$, distobuccal (DB), mesiopalatal (MP) and distopalatal (DP). Contralateral tooth appeared to have normal root canal anatomy.

In case 1, CBCT axial images also showed that the palatal root with a Vertucci type II canal pattern(Fig.1.- i,k,m), (ie, two canal orifices join together and exit as one apical foramen) Mesio-palatal canal is shorter than distopalatal canal and they join in the apical third, whereas the mesiobuccal root showed a Sert and Bayirli type XV canal configuration(Fig. 1-h,j,l) ${ }^{21}$ 
(ie, $\mathrm{MB}^{1}$ and $\mathrm{MB}^{2}$ joined at the middle third of the root and exit in one apical foramen). Whereas in case 2, according to Sert \& Bayirli, this pattern has been described is Type XVIII (3-1) canal configuration (i.e.distobuccal, distolingual and mid-distal joined at the middle third of the root canal and exiting through a single apical foramen)(Fig.2-K).

The instrumentation technique (crown down) used in this case recommend a wide access to the middle and cervical thirds, which facilitated the cleaning of the apical third and the filling of the root canals. Hoen and Pink found $42 \%$ incidence of missed roots or canals in the teeth that needed retreatment) ${ }^{22}$. Hence, it is of utmost importance that all the canals be located and treated during the course of nonsurgical endodontic therapy.

\section{Conclusion}

Information on root canal anatomy along with radiographs is valuable and should always be integrated with a careful clinical examination. The present case report discusses the endodontic management of an unusual case of a maxillary first molar with three roots and five canals and also highlights the role of surgical operating microscope and CBCT scanning as an objective analytic tool to ascertain root canal morphology. The concept of three canals in the maxillary first molar should not be considered as a rule and the clinician should be aware of the incidence of extra canals in the maxillary first molar for achieving predictable success.

\section{References}

[1]. Kulild JC, Peters DD. Incidence and configuration of canal systems in the mesiobuccal root of maxillary first and second molars. J Endod1990;16:311-317

[2]. Buhrley LJ, Barrows MJ, BeGole EA, et al. Effect of magnification on locating the MB2 canal in maxillary molars. J Endod 2002 ;28:324-327

[3]. de Almeida-Gomes F, Maniglia-Ferreira C, Carvalho de Sousa B, et al. Six root canals in maxillary first molar. Oral Surg Oral Med Oral Pathol Oral Radiol Endod 2009 :108: 157-159

[4]. Maggiore F, Jou YT, Kim S. A six-canal maxillary first molar: case report. Int Endod J $2002 ; 35: 486-91$

[5]. Adanir N An unusual maxillary first molar with four roots and six canals:a case report. Aust Dent J 2007; 52:333-335

[6]. B. M. Cleghorn,W. H. Christie, and C. C. S. Dong "Root and root canal morphology of the human permanent maxillary first molar: a literature review". J Endod 2006; 32: 813-821.

[7]. S. Johal, "Unusual maxillary first molar with 2 palatal canals within a single root: a case report," J Can Dent Assoc 2001 ;67: $211-$ 214

[8]. S. Holderrieth and C. R. Gernhardt, "Maxillary molars with morphologic variations of the palatal root canals: a report of four cases,” 2009 J Endod ;35: 1060-1065.

[9]. V. Aggarwal, M. Singla, A. Logani, and N. Shah "Endodontic management of a maxillary first molar with two palatal canals with the aid of spiral computed tomography: a case report," J Endod $2009 ; 35: 137-139$

[10]. M. Deepalakshmi, R. Miglani, R. Indira, and S. Ramachandran "Spiral CT diagnosis and endodontic management of an anatomically variant palatal root with two canals in a maxillary first molar," IJDR 2010;21: 443-445

[11]. Holtzman L. Multiple canal morphology in the maxillary first molar: case reports. Quintessence Int1997;28: 453-455

[12]. Martınez-Berna' A, Ruiz-Badanelli P. Maxillary first molars with six canals. J Endod 1983 ;9: 375-378

[13]. Fabra- Campos CH Unusual root canal anatomy of mandibular first molars. J Endod 1985;11: 568-2

[14]. Caliskan MK, Pehlivan Y, Sepetcioglu F, et al.. Root canal morphology of human permanent teeth in a Turkish population. J Endod $1995 ; 21: 200-204$

[15]. Bond JL, Harwell G, Portell FR. Maxillary first molar with six canals. J Endod $1988 ; 14: 48-49$

[16]. Kottoor, R. Sudha \& N. VelmuruganMiddle distal canal of the mandibular first molar: a case report and literature review Int Endod J 2010; 43: 714-722

[17]. Berthiaume JT .Five canals in lower first molar. The J Mich Dent Assoc $1983 ; 65: 213-217$

[18]. Quackenbush LE .Mandibular molar with three distal root canals. Endod Dent Traumatol 1986; 2: 48-9

[19]. Matherne RP, Angelopoulos C, Kulild JC, Tira D. Use of cone-beam computed tomography to identify root canal systems in vitro. J Endod 2008; 34:87-89.

[20]. Baratto Filho F, Zaitter S, Haragushiku GA, et al. Analysis of the internal anatomy of maxillary first molars by using different methods. J Endod 2009;35:337-342

[21]. Sert S, Bayirli GS Evaluation of root canal configurations of the mandibular and maxillary permanent teeth by gender in the Turkish population. J Endod $2004 ; 30: 391-398$

[22]. 22. Hoen M, Pink, F. Contemporary endodontic retreatments: an analysis based on clinical treatment findings J Endod 2002 ;28(12): 834-836

figure 1:

\section{figures}
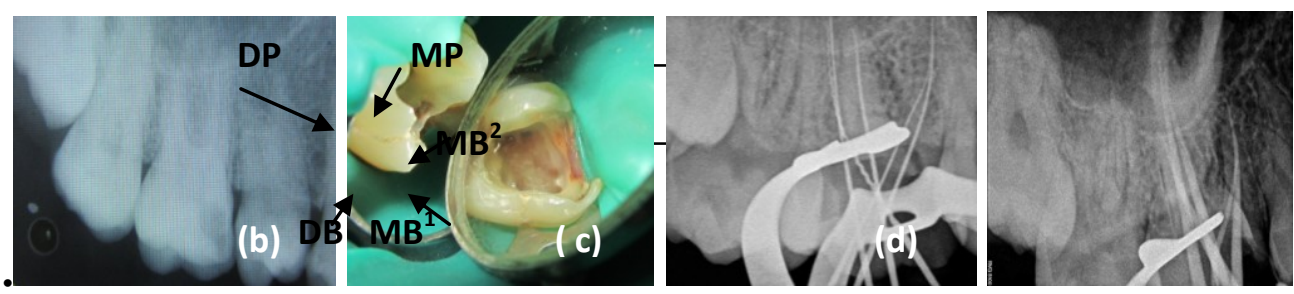


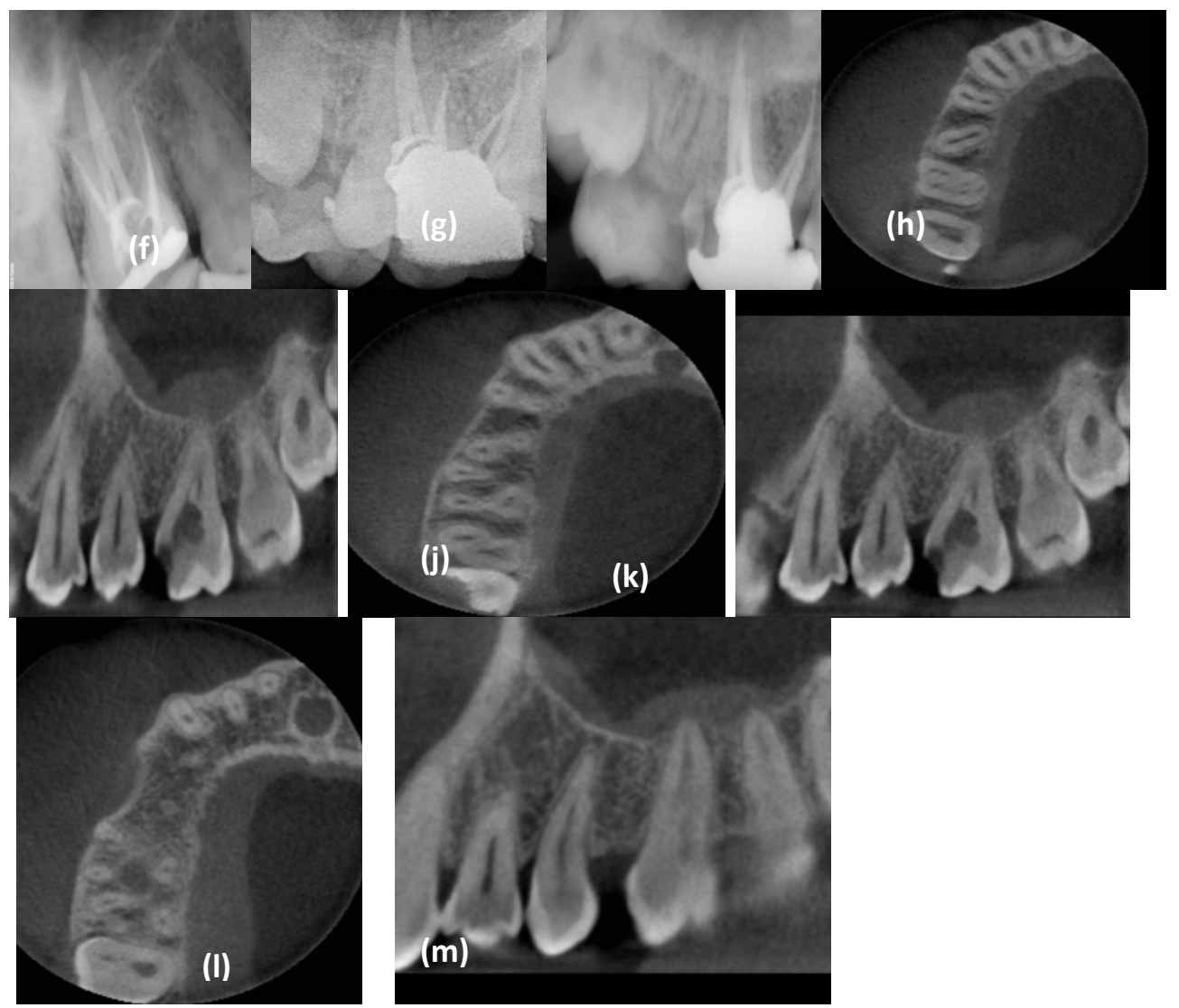

- figure a : preoperative radiograph of 16

- figure $b$ : access opening showing five root canals

- figure $\mathrm{c}$ : working length radiograph of 16 in eccentric angulation

- figure d: master -cone radiograph of 16 in eccentric angulation

- figure e: post obturation radiograph .

- figure f: 6 months follow-up

- figure g: 12 months follow -up

- figure: (h),(i) - cbct images of axial section(g) and sagittal section(h) at coronal level of roots of 16 .

- figure: (j),(k) - cbct images of axial section (i) and sagittal section (j) at middle level of roots of 16.

- figure: (l),(m) - cbct images of axial section (k) and sagittal section (l) at apical level of roots of 16.

Figure 2:

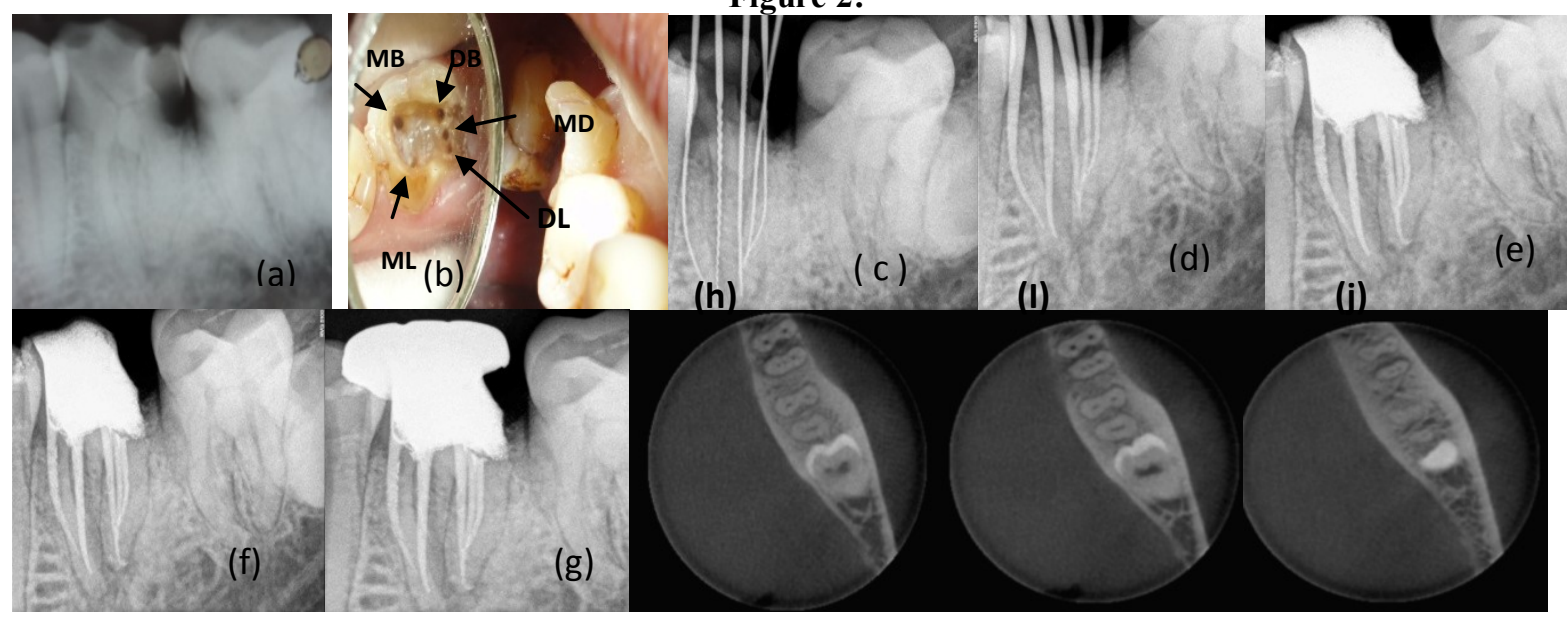




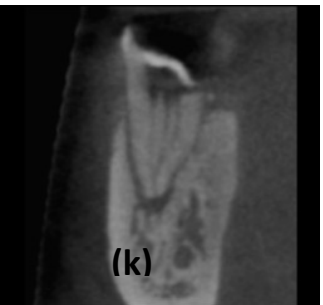

- figure (a) - preoperative radiograph of 36

- figure (b) : access opening showing five root canals

- figure (c) : working length radiograph of 36 in eccentric angulation

- figure (d) : master -cone radiograph of 36 in eccentric angulation

- figure (e): post obturation radiograph

- figure (f) : 6 months follow-up

- figure (g) : 12 months follow-up

- figure: (h) - cbct images of axial section at coronal level of distal root of 36 .

- figure: (i) - cbct images of axial section at middle level of distal root of 36 .

- figure: (j)-cbct images of axial section at apical level of distal root of 36 .

- Figure: (k)- cbct image of sagittal section of distal root of 36 .

\section{Table}

Table 1: Review of case reports of two palatal canals in one palatal root in maxillary first molar.

\begin{tabular}{|llll|}
\hline \multicolumn{1}{|l}{ Investigators } & Study type & Key information \\
\hline$\bullet$ & Johal 2001 & Clinical RCT & 2MB, 1DB, 2P \\
\hline$\bullet$ & $\begin{array}{l}\text { Holderrieth and Gernhardt } \\
2009\end{array}$ & Clinical RCT & 2 Cases: 2MB, 1DB,2P \\
\hline$\bullet$ & Aggarwal et al. 2009 & Spiral CT & 1MB, 1DB, 2P \\
\hline$\bullet$ & Deepalakshmi et al. 2009 & Spiral CT & 2MB, 1DB, 2P \\
\hline & Holtzman (1997) & Clinical RCT & 2MB, 1DB, 2P \\
\hline & & \\
\hline
\end{tabular}

